\title{
Acculturation, Reading Level, and Neuropsychological Test Performance Among African American Elders
}

\author{
Jennifer J. Manly, Desiree A. Byrd, and Pegah Touradji \\ Cognitive Neuroscience Division, Taub Institute for Research \\ on Alzheimer's Disease and the Aging Brain, Department of Neurology, \\ Columbia University College of Physicians and Surgeons, New York, New York, USA

\begin{abstract}
Yaakov Stern
Cognitive Neuroscience Division, Taub Institute for Research

on Alzheimer's Disease and the Aging Brain, Department of Neurology,

Columbia University College of Physicians and Surgeons, New York, New York, USA, and Department of Psychiatry, Columbia University College of Physicians and Surgeons, New York, New York, USA
\end{abstract}

\begin{abstract}
The independent effects of cultural and educational experience on neuropsychological test performance were examined among 503 nondemented African Americans ages 65 and older. Measures of cultural experience (acculturation) and quality of education (reading level) were administered. Reading level was the most influential predictor of cognitive test performance, even after accounting for age, sex, years of education, and acculturation level. Age had small but significant unique effects on most measures, especially word list learning. Years of education had independent effects on measures of verbal abstraction, fluency, and figure matching. More acculturated African Americans obtained higher scores on most measures; however, after accounting for age, years of education, sex, and reading level, the effect of acculturation was diminished. The results suggest that quality of education and cultural experience influence how older African Americans approach neuropsychological tasks; therefore, adjustment for these variables may improve specificity of neuropsychological measures.
\end{abstract}

Key words: quality of education, acculturation

Most studies of ethnic group differences in performance on intelligence, screening, and other neuropsychological measures have shown that discrepancies between scores of African Americans and Whites persist, despite matching groups on variables such as age, edu-

This research was supported by federal grants AG16206 (J. Manly), AG07232 (R. Mayeux), the Alzheimer's Association, and the New York City Speakers Fund for Biomedical Research-Toward the Science of Patient Care. The authors thank Rosann Costa for her help with data management and Judes Fleurimont, Maria Gonzalez-Diaz, Cherita McDowell, Danurys Sanchez, Stacey Tuchin, and Wizdom Powell for their assistance with scheduling and interviewing participants.

Pegah Touradji is completing her postdoctorial fellowship at James A. Haley Veterans Hospital in Tampa, Florida.

Requests for reprints should be sent to Jennifer Manly, G.H. Sergievsky Center, 630 West 168 th Street, P \& S Box 16, New York, NY 10032. E-mail: jjm71@ columbia.edu cation, sex, and income (reviewed in Manly \& Jacobs, 2001; Nabors, Evans, \& Strickland, 2000). These discrepancies attenuate the specificity of neuropsychological tests such that cognitively normal African Americans are more likely than Whites to be misdiagnosed as cognitively impaired (Ford-Booker et al., 1993; Klusman, Moulton, Hornbostle, Picano, \& Beattie, 1991; Manly et al., 1998b; Stern et al., 1992; Welsh et al., 1995). Many investigators have concluded that separate neuropsychological test norms for African Americans and other ethnic groups are the solution to this problem (Ardila, 1995; Nabors et al., 2000; Wong, Strickland, Fletcher-Janzen, Ardila, \& Reynolds, 2000).

Use of separate ethnic group norms most likely will reduce misdiagnosis of cognitive impairment among African Americans; however, there are significant disadvantages to this approach. Primarily, the use of sep- 
arate norms may leave ethnic differences in test performance unexplained, unexamined, and thus not understood. Many authors (Helms, 1992; Neisser et al., 1996) have described how genetic or biological factors are often invoked to account for these unexplained differences. In contrast to the biological/genetic hypothesis, researchers in the 1970 s began to propose that, because intelligence tests and other achievement measures incorrectly assumed a high level of literacy and equivalent school experiences, the tests were culturally biased against ethnic minorities and had unacceptable reliability and validity when used in these groups (Hilliard, 1979; Williams, 1971, 1974; van de Vijver, 1997). Helms argued that persisting test score discrepancies between African Americans and Whites reflect poor functional equivalence of cognitive tasks in the two ethnic groups. That is, measures developed in the majority culture may not assess the same cognitive ability in the same way when applied to African Americans. Early challenges to the biological/genetic hypotheses also focused on the ability of socioeconomic differences to explain ethnic differences in cognitive test performance. One of the first studies on quality of education and cognitive test performance showed that African American Army recruits schooled in northern states obtained higher average scores on the Army Alpha test than Whites schooled in southern states; furthermore, African Americans' scores increased for every year they stayed in northern cities (Klineberg, 1935). Still other investigators focused on measurement issues and held that, once socioeconomic variables were properly measured and accounted for, ethnic differences in IQ would disappear (Mercer, 1974).

Another major drawback underlying separate ethnic group norms is the assumption embedded in racial and ethnic classifications. Use of norms separated on the basis of race assumes that racial classifications are consistent and scientific. Because "Black," "African American," and "White" are social-political classifications as opposed to scientifically or genetically defined categories (Harrison, 1995; Smedley, 1999; D. Y. Wilkinson \& King, 1987), it is unclear who should be included in normative studies for African Americans and to whom these norms should be applied. As the number of biracial and multiracial Americans increases, neuropsychologists would have to address how ethnic group norms would be applied to those with mixed heritage for whom traditional racial classifications are unclear or inappropriate. These complicated issues highlight the advantages of deconstructing race into the consistent, meaningful, and predictive variables that under- lie the relationship between race and cognitive test performance.

As early as 1935, studies such as Klineberg's (1935) work with African American and White Army recruits demonstrated that specification of experiential, attitudinal, or behavioral variables that distinguish those belonging to different ethnic and racial groups and that also vary among people within an ethnic group may allow investigators to understand the underlying reasons for ethnic group differences in cognitive test performance. There is tremendous diversity in geographic, economic, and educational experiences, as well as level of exposure to majority culture, among African Americans. Current racial and ethnic classifications ignore this diversity. Our research group has begun to identify these within-group cultural factors, to measure them, and to explore their relationship to cognitive test performance. This investigational approach may illuminate factors that can explain ethnic group differences on cognitive tests. It also can inform us in the future regarding the development of measures of cognitive abilities that are salient within African American culture. The effect of these cultural and educational factors on cognitive test performance must be well understood before the development of "culture-fair" measures.

Level of acculturation was the first variable our research team used to operationalize within-group cultural variability. Previous studies have identified ideologies, beliefs, expectations, and attitudes as important components of acculturation, as well as cognitive and behavioral characteristics such as language and cognitive style (Berry, 1976; Moyerman \& Forman, 1992; Negy \& Woods, 1992; Padilla, 1980). Acculturation has traditionally been measured among immigrant groups such as Hispanics and Asian Americans; however, in 1994, Landrine and Klonoff (1994) reported the development of a reliable and valid measure of African American acculturation. This scale assesses traditional childhood experiences; religious beliefs and practices; preferences for African American music, media, and people; and preparation and consumption of traditional foods. When acculturation is measured, the association of cultural experience with cognitive test performance can be assessed, and hypotheses regarding test performance among people with lifestyles that are very dissimilar to the majority culture can be tested.

Previous research on Hispanic groups has shown a relationship between acculturation and performance on selected tests of the Halstead-Reitan Battery among college students (Arnold, Montgomery, Castaneda, \& Longoria, 1994), as well as a relationship between years in the United States (a strong correlate of accul- 
turation level) and perseverative errors on the Wisconsin Card Sorting Test (Artiola i Fortuny, Heaton, \& Hermosillo, 1998). Three studies have explored the relationship of African American acculturation to cognitive test performance. Manly and colleagues (Manly et al., 1998) found that, among neurologically intact African Americans between the ages of 20 and 65, those who were less acculturated (more traditional) obtained lower scores on the Wechsler Adult Intelligence Scale-Revised (WAIS-R) Information subtest and the Boston Naming Test than more acculturated African Americans. Also in this study, differences in neuropsychological test scores between age, education, and disease-stage-matched HIV-positive African Americans and Whites were eliminated after controlling for acculturation level. Among elderly African Americans living in Jacksonville, Florida, acculturation accounted for a significant amount of variance in Verbal IQ (as measured by the WAIS-R), Boston Naming Test, and delayed recall of stories from the Wechsler Memory Scale-Revised (Lucas, 1998). A recent study found that, among African American traumatic brain injury patients, those who were less acculturated obtained lower scores on a neuropsychological test battery overall and specifically on the Grooved Pegboard and WAIS-R Block Design and achieved a lower number of categories on the Wisconsin Card Sorting Test (Kennepohl, Shore, Nabors, \& Hanks, in press). A preliminary study among elderly, nondemented African American residents of northern Manhattan revealed that those who were more traditional (less acculturated), as assessed by the African American Acculturation Scale (AAAS), obtained lower scores on measures of figure memory, naming, repetition, and figure matching (Manly et al., 1998a). Taken together, investigations of acculturation suggest that there are cultural differences within elders of the same ethnicity that relate to neuropsychological measures of verbal skills, executive function, and psychomotor speed and that accounting for acculturation (in addition to age, years of education, and sex) may help improve the ability of certain neuropsychological tests to detect subtle impairment.

Schooling is another aspect of cultural experience that has been proven to have significant effects on neuropsychological test performance, regardless of race or ethnicity (Adams, Boake, \& Crain, 1982; Heaton, Grant, \& Matthews, 1986; Lezak, 1995). However, in the United States, there is a great deal of discordance between years of education and quality of education; this is especially true among African Americans. Factors such as whether African Americans attended school before or after the Supreme Court's 1954 Brown v. Board of Education decision banning segregation in public schools, unequal distribution of educational funds, variable teacher education, shorter length of school year, and lower attendance because of required work had a profound effect on the quality of education received by African Americans (Anderson, 1988; Margo, 1990). These variables are among the multiple factors that have been found to explain the differences between African Americans and Whites on achievement measures and other outcomes such as wage earnings (Hanushek, 1989; Hedges, Laine, \& Greenwald, 1994; O'Neill, 1990). Previous studies have demonstrated that African Americans had reading skills that were significantly below their self-reported education level (Albert \& Teresi, 1999; Baker, Johnson, Velli, \& Wiley, 1996; Manly, Jacobs, Touradji, Small, \& Stern, 2002). Therefore, disparate school experiences and resulting different bases of problem-solving strategies, knowledge, familiarity, and practice could explain why some African Americans obtain lower scores on cognitive measures even after controlling for years of education. Statistical control for years of education may be an inadequate or inappropriate method for equating racial groups on educational experience because quality of education differs so dramatically between African Americans and Whites, as well as within African Americans (e.g., depending on whether they were educated in segregated or integrated schools or in rural or urban settings; Kaufman, Cooper, \& McGee, 1997; Loewenstein, Arguelles, Arguelles, \& Linn-Fuentes, 1994).

Our research group recently reported a study that sought to determine whether discrepancies in quality of education could explain differences in cognitive test scores between African American and White elders matched on years of education (Manly et al., 2002). A comprehensive neuropsychological battery was administered to a sample of nondemented African American and non-Hispanic White participants in an epidemiological study of normal aging and dementia in the northern Manhattan community. The Reading Recognition subtest from the Wide Range Achievement Test-Version 3 (WRAT-3) was used as an estimate of quality of education. African American elders obtained significantly lower scores than Whites on measures of word list learning and memory, figure memory, abstract reasoning, fluency, and visuospatial skill, even though the groups were matched on years of education. However, after adjusting the scores for WRAT-3 reading score, the overall effect of race was greatly reduced, and racial differences on all tests (except category fluency and a drawing measure) became nonsignificant. 
Reading score also attenuated the effect of race after accounting for an estimate of test wiseness. This finding suggests that years of education is an inadequate measure of the educational experience among multicultural elders and that adjusting for quality of education may improve the specificity of certain neuropsychological measures across racial groups.

The investigation reported here was an attempt to determine the role of age, sex, years of education, quality of education (as operationalized by reading level), and acculturation on neuropsychological test performance among African American elders. Our previous research (Manly et al., 1999; Manly et al., 2002; Manly et al., 1998 ) indicated that each of these variables would account for a significant amount of variance on performance on cognitive measures that tapped both verbal and nonverbal functions. However, because acculturation and reading level had not yet been measured in the same sample, it was unclear whether each would contribute uniquely to the prediction of cognitive test performance. Nevertheless, it was expected that elders with a high quality of education (and thus high reading levels) would obtain higher scores on neuropsychological measures of nonverbal memory, abstraction, naming, letter fluency, repetition, comprehension, and visuospatial skill than elders with a low quality of education and reading levels. Furthermore, it was hypothesized that reading level would be the strongest predictor of these cognitive test scores and thus would account for significant, unique variance in test scores when years of education, age, sex, and acculturation were entered into the model. With regard to cultural experience, it was expected that more acculturated African American elders would obtain higher scores on measures of figure recognition, verbal abstraction, naming, and figure matching than less acculturated elders. Acculturation was predicted to be a significant predictor of performance on these measures, albeit a weaker predictor than reading level.

\section{Method}

\section{Research Participants}

The sample was selected from participants in the Washington Heights-Inwood Columbia Aging Project (WHICAP), a community-based, epidemiological study of dementia in the ethnically diverse neighborhoods of northern Manhattan, New York. WHICAP longitudinally follows a sample of elderly Medicare recipients residing in selected census tracts of Washington/Hamilton Heights and Inwood. The population from which participants were drawn consists of people from several different countries of origin and represents three broadly defined ethnic categories (i.e., Hispanic, African American, and non-Hispanic White).

Inclusion and exclusion criteria. All potential participants were aged 65 and older and completed the neuropsychological battery in English. Participants were included if they self-identified their race as Black/African American and their "ethnicity" as non-Hispanic according to the 1997 revisions to the Office of Management and Budget's standards for data on race and ethnicity, which was used by the 2000 U.S. Census. Approximately $10 \%$ of WHICAP participants who described themselves as non-Hispanic Black were born in Jamaica, other Caribbean Islands such as Haiti, or Central America; the sample reported here was limited to African American elders born in the United States and for whom English was their first and primary language. Potential participants were excluded if they had a history of Parkinson's disease, stroke, head injury with loss of consciousness, alcohol or substance abuse, history of mental illness such as schizophrenia, or current major depression. Only WHICAP participants who showed no neurological or functional signs of dementia were included in the analyses reported here. This determination was made on the basis of a physician's clinical examination, which included a rating of daily functioning (see Procedure later in this article). The physician's diagnosis was used as a "gold standard" for the absence of dementia because the neurological assessment was made independent of the participant's performance on the neuropsychological battery.

Medical evaluation. A physician recorded medical history and medications in a semistructured format. Neurological and brief physical examinations were performed, including assessment of extrapyramidal signs. Functional status was measured using Part 1 of the Blessed Dementia Rating Scale (Blessed, Tomlinson, \& Roth, 1968) and the Schwab and England rating scale of activities of daily living (Boller, Mizutani, Roessmann, $\&$ Gambetti, 1980). From this information, the physician determined whether the participant met criteria for delirium or dementia using the Diagnostic and Statistical Manual of Mental Disorders 3rd. ed., Revised (American Psychiatric Association, 1987) criteria.

\section{Procedure}

Neuropsychological battery. The evaluation included measures of learning, memory, orientation, ab- 
stract reasoning, language, and visuospatial ability. Specific ability areas and tests administered included verbal list learning and memory using the Selective Reminding Test (SRT; Buschke \& Fuld, 1974); nonverbal memory from the multiple-choice version of the Benton Visual Retention Test (BVRT; Benton, 1955); orientation items from the Mini Mental State Examination (MMSE; Folstein, Folstein, \& McHugh, 1975); verbal reasoning using the Similarities subtest of the WAIS-R (Wechsler, 1981); nonverbal reasoning from the Identities and Oddities subtest of the Mattis Dementia Rating Scale (DRS; Mattis, 1976); naming using a 15-item version of the Boston Naming Test (Kaplan, Goodglass, \& Weintraub, 1983); letter fluency from the Controlled Word Association Test (Benton \& Hamsher, 1976); category fluency using procedures from the Boston Diagnostic Aphasia Examination (BDAE) and the categories of animals, food, and clothing (Goodglass \& Kaplan, 1983); repetition of high-frequency phrases from the BDAE (Goodglass et al., 1983); auditory comprehension of the first 6 items of the Complex Ideational Material subtest of the BDAE (Goodglass et al., 1983); visuoconstruction using the Rosen Drawing Test (Rosen, 1981); and visuoperceptual skills as assessed by multiple-choice matching of figures from the BVRT (Benton, 1955).

Reading level. Reading level was measured using the Reading Recognition subtest from the WRAT-3 (G. S. Wilkinson, 1993). Participants were asked to name letters and pronounce words out of context. The words are listed in order of decreasing familiarity and increasing phonological complexity. Consistent with the standard instructions for administration, a basal of 5 correct and a ceiling of 10 incorrect was used.

Acculturation. Each participant's level of acculturation was measured using the short form of the AAAS (Landrine \& Klonoff, 1994, 1995). Each of the 33 items assesses traditions, values, beliefs, assumptions, and practices found in African American culture. The short-form AAAS measures acculturation across 10 dimensions: preference for African American music, arts, and people; religious beliefs and practices; traditional foods; traditional childhood experiences; superstitions; interracial attitudes/cultural mistrust; falling out (knowledge and experience of a folk disorder); traditional games; and family values. The four AAAS subscales that accounted for the most variance in the Landrine and Klonoff (1995) standardization sample were preferences (e.g., "Most of the music I lis- ten to is by Black artists"); foods and food practices (e.g., "Sometimes, I cook ham hocks"); religious beliefs and practices (e.g., "I am currently a member of a Black church"); and traditional childhood experiences (e.g., "I grew up in a mostly Black neighborhood"). Landrine and Klonoff (1995) found that the AAAS-33 could distinguish well between African Americans and non-African Americans, had acceptable concurrent validity, split-half reliability of $r=.77$, and an internal consistency reliability (Cronbach's alpha) of $r=.81$ among African Americans. Scores on the subscales were not significantly related to social class, education, or social class of origin in the original standardization sample. In its original version, the AAAS is a self-administered, paper-and-pencil survey. To accommodate older participants who had poor vision or low levels of literacy, the measure was slightly modified from the published version such that the scale was administered in a face-to-face interview and the original Likert scale was simplified. In this slightly modified version, participants chose one of the following responses to each item: "I disagree/This is not at all true of me," "Sort of agree/This is sort of true of me," or "I strongly agree/This is definitely true of me." Our modified version has an internal consistency reliability (Cronbach's alpha) of .71 and a Guttman split-half reliability of $r=$ .61 (Powell, Tuchin, Touradji, \& Manly, 2000). In the analyses presented here, the summary score from the AAAS was used to represent acculturation level.

\section{Statistical Methods}

Simple bivariate correlation analyses and $t$ tests were used to determine the strength of the relationships among the predictors: age, sex, years of education, acculturation (total score on the AAAS), and WRAT-3 reading level.

The next set of analyses used simple regression models to determine the relationships between each of the five predictor variables (age, sex, years of education, total score on the AAAS, and WRAT-3 reading level) and performance on each of the neuropsychological tests. The 13 measures used as dependent variables were total raw scores for immediate recall and delayed recall from the SRT, BVRT matching and recognition score, items correct on the MMSE Orientation, 15-item Boston Naming, BDAE repetition, BDAE comprehension, Mattis Identities and Oddities, number correct on the Rosen Drawing Test, raw score on WAIS-R Similarities, and mean number of words generated over three 60-sec trials for category and letter fluency. Each of these 13 dependent variables was predicted by each 
of the 5 dependent variables, which were entered into the equation alone. Effect size is reported as the proportion of variance in each test score accounted for by the predictor variable.

Finally, multiple regression was used to determine the independent contributions of age, sex, years of education, acculturation level, and reading score to the prediction of each neuropsychological test score. In each regression equation, age, years of education, sex, acculturation, and reading level were entered together as predictors of neuropsychological test performance. The partial regression coefficients for each of the predictors were examined to determine which variables accounted for unique and significant amounts of variance in each cognitive test score. Alpha for all analyses was set at $p<$ .01 to strike a balance between the likelihood of committing Type I and Type II errors.

\section{Results}

\section{Sample Characteristics}

A total of 554 nondemented, English-speaking, American-born, non-Hispanic Black WHICAP participants had complete neuropsychological evaluations, WRAT-3 data, and neurological exams. Fifty-one participants were excluded because of a history of stroke $(n=17)$, head injury $(n=8)$, drug or alcohol abuse $(n=$ $5)$, or psychiatric history $(n=23)$. Some patients had more than one exclusionary condition.

The remaining 503 African American elders had a mean age of 76.2 years $(S D=6.1)$, and $75 \%$ were women. Average years of education was 11.4 years $(S D$ $=3.7)$ and ranged from 0 to 20 years. WRAT -3 reading subtest scores ranged from 13 (0-third-grade reading level) to 56 (post-high school reading level), with a mean of $42.2(S D=8.1)$.

\section{Correlations Between Demographics, Acculturation, and Reading Level}

The correlations between the primary independent variables are shown in Table 1 . The correlations between these variables, although significant, indicate that the variables reflect constructs that are not multicollinear. Older African Americans had fewer years of education relative to younger African Americans. As expected, African Americans with more years of education had higher scores on the WRAT- 3 reading subtest and were more acculturated. Higher scores on the WRAT-3 reading subtest were associated with reports of less traditional (more acculturated) lifestyle as re-
Table 1. Correlations Between Independent Variables

\begin{tabular}{llcc}
\hline & Age & $\begin{array}{c}\text { Years of } \\
\text { Education }\end{array}$ & AAAS Total \\
\hline Age & 1.00 & & \\
Years of education & $-.15^{*}$ & 1.00 & \\
AAAS total & -.07 & $-.24^{*}$ & 1.00 \\
WRAT-3 reading & -.08 & $.59^{*}$ & $-.28^{*}$ \\
\hline
\end{tabular}

Note: AAAS = African American Acculturation Scale; WRAT-3 = Wide-Range Achievement Test-Version 3.

${ }^{*} p<.001$.

ported on the AAAS. $T$ tests showed that elderly African American women in this sample were significantly older than elderly African American men, $t(501)=$ $3.77, p<.001$.

\section{Demographics, Acculturation, Reading Level, and Neuropsychological Test Performance}

Table 2 shows the proportion of variance accounted for by each of the predictor variables in simple regression equations predicting each of the neuropsychological test scores. Sex was not a significant predictor of performance on any of the measures, accounting for no more than $0.5 \%$ of variance in any of the scores. Age accounted for $1 \%$ to $11 \%$ of variance in neuropsychological test scores, with its strongest effects on SRT total recall score. Elders with fewer years of school obtained lower scores on the measures; the effect size of years of education ranged from $2 \%$ to $32 \%$. Acculturation was a significant but weak predictor of test scores, accounting for no more than $6 \%$ of variance on any measure. Finally, WRAT-3 reading score had strong, positive relationships with performance, accounting for $3 \%$ to $40 \%$ of the variance in test scores.

Multiple regression analyses revealed that reading level was the strongest independent predictor of most measures in the neuropsychological battery $(p<.001$ for all). Among the independent variables, reading level accounted for the largest proportion of unique variance on all but 5 of the 13 variables: SRT total and delayed recall, Mattis DRS Identities and Oddities, category fluency, and BVRT matching. In contrast, although years of education had an independent effect on 6 of the measures, it did not uniquely contribute to the prediction of delayed recall of a word list, orientation, nonverbal abstract reasoning, naming, repetition, comprehension, or drawing.

Age was a significant, independent predictor of scores on all measures except orientation, repetition, and comprehension $(p<.005$ for all). Acculturation 
Table 2. Proportion of Variance $\left(\mathrm{R}^{2}\right)$ in Neuropsychological Test Scores Accounted for by Demographics, Acculturation, and Reading Level

\begin{tabular}{|c|c|c|c|c|c|}
\hline Test & Sex & Age & Years of Education & Acculturation & WRAT-3 Reading \\
\hline \multicolumn{6}{|l|}{ Learning/memory } \\
\hline SRT total recall & .001 & .119 & .166 & .025 & .189 \\
\hline SRT delayed recall & .001 & .070 & .091 & $.011^{\mathrm{b}}$ & .105 \\
\hline BVRT recognition memory & .004 & .059 & .156 & .029 & .211 \\
\hline \multicolumn{6}{|l|}{ Orientation } \\
\hline MMSE orientation & .004 & $.012^{\mathrm{b}}$ & .020 & $.002^{\mathrm{b}}$ & .033 \\
\hline \multicolumn{6}{|l|}{ Abstract reasoning } \\
\hline WAIS-R similarities raw & .005 & .062 & .319 & .060 & .386 \\
\hline DRS identities and oddities & .005 & .045 & .077 & $.003^{\mathrm{b}}$ & .088 \\
\hline \multicolumn{6}{|l|}{ Language } \\
\hline Boston Naming & .002 & .025 & .148 & .048 & .316 \\
\hline Letter fluency & .002 & .046 & .216 & .030 & .401 \\
\hline Category fluency & .000 & .070 & .184 & .028 & .184 \\
\hline BDAE repetition & .004 & $.010^{\mathrm{b}}$ & .057 & .028 & .095 \\
\hline BDAE comprehension & .003 & $.012^{\mathrm{b}}$ & .096 & .017 & .137 \\
\hline \multicolumn{6}{|l|}{ Visuospatial ability } \\
\hline Rosen Drawing & .001 & .036 & .071 & .039 & .116 \\
\hline BVRT matching & .001 & .058 & .139 & .038 & .156 \\
\hline
\end{tabular}

Note: $R^{2}$ is the proportion of variance in neuropsychological test score accounted for by the independent variable in a simple linear regression. WRAT $-3=$ Wide Range Achievement Test-Version 3; SRT = Selective Reminding Test; BVRT = Benton Visual Retention Test; MMSE = Mini Mental State Examination; WAIS-R = Wechsler Adult Intelligence Scale-Revised; DRS = Dementia Rating Scale; BDAE = Boston Diagnostic Aphasia Examination. All values shown had a $p$ value less than .01 with the following exceptions.

aNone of the $R^{2}$ values for the effect of sex were significant below the .01 level. bThese values did not reach significance.

contributed significantly to the prediction of scores on only Rosen Drawing; however, there was a trend for more acculturated African American elders to obtain higher scores on the WAIS-R Similarities subtest $\left(s r^{2}=\right.$ $.01, p=.03)$ and delayed figure recognition $\left(s r^{2}=.01, p\right.$ $=.02$ ) than more traditional (less acculturated) elders. Sex was not a significant unique predictor of performance on any of the cognitive measures.

\section{Discussion}

The results show that, among African American elders, age, years of education, acculturation level, and reading ability have significant, independent effects on neuropsychological test scores across several cognitive domains. The magnitude of the association between reading level and test performance was greatest on measures of verbal abstraction, naming, and phonemic fluency. Furthermore, reading level was consistently a significant unique predictor of test performance across cognitive domains. Older African Americans obtained significantly lower scores on several measures, especially verbal word list learning; however, the independent effect of age on test score was weak relative to the association between reading level and test score. Elders with fewer years of school obtained lower scores on all measures; but again, in the context of the other demo- graphic variables such as acculturation and reading level, the association of years of education with test performance was diminished. Finally, acculturation level was a unique predictor of only drawing skill. These findings support the use of within-group cultural and educational variables to adjust expectations of test performance among African American elders.

Hypotheses regarding quality of education (as operationalized by reading level) were based on our previous work with elders with no formal education (Manly et al., 1999), as well as a study that used the WRAT-3 to explain differences in test performance between African American and White elders (Manly et al., 2002). These hypotheses were partially confirmed. African Americans with lower reading levels obtained lower scores on measures of figure memory, verbal abstraction, naming, letter fluency, comprehension, phrase repetition, and visuospatial skill; these effects were independent of acculturation, age, years of education, and sex. However, contrary to our original hypotheses, reading level also had a significant unique effect on learning and delayed recall scores from the SRT, orientation, a measure of nonverbal abstraction (Identities and Oddities from the DRS), as well as words generated within a particular category (animals, foods, and clothing). Nevertheless, the effect sizes of reading level for these measures ( $2 \%$ to $7 \%$ of the variance of the test scores uniquely accounted for by WRAT-3) were much 
smaller than the effect sizes of reading level for other measures such as verbal abstraction, naming, and letter fluency (ranging from 5\% to 26\%).

These results add to the literature, suggesting that reading level is a more sensitive proxy for educational experience than years of education among African American elders. However, educational quality is only one of many variables that may influence reading recognition scores, including general (native) cognitive ability, reading experience obtained through access to books in the home or as a result of occupational demands, and test wiseness. Future study will explore the relationship of WRAT-3 reading score to other measures of educational quality such as per-student expenditures, teacher salaries, and length of school year. Regardless of the abilities and experiences that determine reading level, this skill appears to be the strongest predictor of cognitive test performance among African American elders.

It was hypothesized that acculturation level would be a unique predictor of performance on certain cognitive measures; however, this hypothesis was largely unsupported by the data. African American elders who were more acculturated obtained higher scores than traditional African American elders on all measures except delayed recall of a word list, orientation, and nonverbal abstraction. However, once other demographic variables and reading level were entered into the model, the unique effect of acculturation was significant only for Rosen Drawing, in which AAAS score accounted for only $2 \%$ of the variance.

The association of cognitive test performance with acculturation level and reading skill could be explained by other cognitive and noncognitive factors that have a direct influence on test performance. For example, acculturation level may reflect the salience that a particular task has in everyday life. Traditional cognitive measures are based within a dominant culture that emphasizes individualism, detail, and speeded performance, whereas traditional African Americans are more likely to ascribe to a belief system that is spiritually based and holistic and that emphasizes interpersonal relationships (Asante, 1990; Boykin, Jagers, Ellison, \& Albury, 1997; Shade, 1991). Less acculturated elders, as well as African Americans who attended poorly funded, segregated schools, may not be as "testwise" or as proficient in the implicit and explicit language of neuropsychological assessment. Acculturation and reading level also may reflect the influence of racial socialization on motivation or attitude toward testing. For example, African American children with higher levels of cultural mistrust obtained lower scores on cognitive measures when assessed by a White examiner (Terrell, Terrell, \& Taylor, 1981).

The complete range of cognitive skills and learning strategies of ethnic minorities may not be adequately tapped by standard cognitive tasks. Perhaps traditional neuropsychological tests simply do not elicit the full potential of all African Americans and other ethnic minority groups. Assessment of these yet-unmeasured strengths could be the best way to detect subtle neurocognitive dysfunction among ethnic minorities. Evidence that supports this possibility is derived from studies demonstrating that, when test stimuli are more culturally pertinent to the experiences of African Americans, performance improves (Hayles, 1991). Prior research indicates that African Americans obtain higher scaled scores on measures of divergent thinking or creativity than on traditional measures of general information or verbal abstraction (Price-Williams \& Ramirez, 1977; Torrance, 1971). In addition, some research shows that, in contrast to reports of lower African American performance on memory tests with verbal and figural stimuli, African Americans obtain higher scores on measures of facial recognition (both White and Black faces) than Whites (Barkowitz \& Brigham, 1982; Golby, Gabrieli, Chiao, \& Eberhardt, 2001), suggesting increased salience or experience with human face processing among African Americans. These studies point to several alternative ways in which neuropsychological measures can be used to assess cognitive abilities that are salient within African American culture.

Studies of the effect of within-group cultural and educational factors on test performance can serve as hypothesis generators for in-depth study of the effect of these variables on specific cognitive functions. Once we understand the nature of the relationship between cultural and educational variables and test performance, we may be able to more successfully develop tests that are specific and sensitive to neurocognitive impairment among African Americans.

\section{References}

Adams, R. L., Boake, C., \& Crain, C. (1982). Bias in a neuropsychological test classification related to age, education and ethnicity. Journal of Consulting and Clinical Psychology, 50, 143-145.

Albert, S. M., \& Teresi, J. A. (1999). Reading ability, education, and cognitive status assessment among older adults in Harlem, New York City. American Journal of Public Health, 89, 95-97.

American Psychiatric Association. (1987). Diagnostic and Statistical Manual of Mental Disorders (3rd ed., revised). Washington, DC: Author. 
Anderson, J. D. (1988). The education of Blacks in the South, 1860-1935. Chapel Hill: University of North Carolina Press.

Ardila, A. (1995). Directions of research in cross-cultural neuropsychology. Journal of Clinical and Experimental Neuropsychology, I7, 143-150.

Arnold, B. R., Montgomery, G. T., Castaneda, I., \& Longoria, R. (1994). Acculturation and performance of Hispanics on selected Halstead-Reitan neuropsychological tests. Assessment, 1, 239-248.

Artiola i Fortuny, L., Heaton, R. K., \& Hermosillo, D. (1998). Neuropsychological comparisons of Spanish-speaking participants from the U.S.-Mexico border region versus Spain. Journal of the International Neuropsychological Society, 4 , 363-379.

Asante, M. K., \& Asante, A. K. W. (1990). African culture and the rhythms of unity. Trenton, NJ: Africa World Press.

Baker, F. M., Johnson, J. T., Velli, S. A., \& Wiley, C. (1996). Congruence between education and reading levels of older persons. Psychiatric Services, 47, 194-196.

Barkowitz, P., \& Brigham, J. C. (1982). Recognition of faces: Own-race, incentive, and time-delay. Journal of Applied Social Psychology, 12, 225-268.

Benton, A. L. (1955). The Visual Retention Test. New York: Psychological Corporation.

Benton, A. L., \& Hamsher, K. D. (1976). Multilingual aphasia examination. Iowa City: University of Iowa Press.

Berry, J. W. (1976). Human ecology and cognitive style. New York: Sage-Halsted.

Blessed, G., Tomlinson, B. E., \& Roth, M. (1968). The association between quantitative measures of senile change in the cerebral grey matter of elderly subjects. British Journal of Psychology, 114, 797-811.

Boller, F., Mizutani, T., Roessmann, U., \& Gambetti, P. (1980). Parkinson's disease, dementia, and Alzheimer's disease: Clinicopathological correlations. Annals of Neurology, 1, 329-335.

Boykin, W., Jagers, R. J., Ellison, C. M., \& Albury, A. (1997). Communalism: Conceptualization and measurement of an Afrocultural social orientation. Journal of Black Studies, 27, 409-418.

Buschke, H., \& Fuld, P. A. (1974). Evaluating storage, retention, and retrieval in disordered memory and learning. Neurology, 24, 1019-1025.

Folstein, M. F., Folstein, S. E., \& McHugh, P. R. (1975). "MiniMental State": A practical method for grading the cognitive state of patients for the clinician. Journal of Psychiatric Research, 12, 189-198.

Ford-Booker, P., Campbell, A., Combs, S., Lewis, S., Ocampo, C., Brown, A., et al. (1993). The predictive accuracy of neuropsychological tests in a normal population of African Americans. Journal of Clinical and Experimental Neuropsychology, 15, 64.

Golby, A. J., Gabrieli, J. D., Chiao, J. Y., \& Eberhardt, J. L. (2001). Differential responses in the fusiform region to same-race and other-race faces. Nature Neuroscience, 4, 845-850.

Goodglass, H., \& Kaplan, E. (1983). The assessment of aphasia and related disorders (2nd ed.). Philadelphia: Lea \& Febiger.

Hanushek, E. (1989). The impact of differential expenditures on school performance. Educational Researcher, 18(4), 45-51.

Harrison, F. V. (1995). The persistent power of "race" in the cultural and political economy of racism. Annual Review of Anthropology, 24, 47-74.
Hayles, V. R. (1991). African American strengths: A survey of empirical findings. In R. L. Jones (Ed.), Black psychology (3rd ed., pp. 379-400). Berkeley, CA: Cobb \& Henry Publishers.

Heaton, R. K., Grant, I., \& Matthews, C. G. (1986). Differences in neuropsychological test performance associated with age, education, and sex. In I. Grant \& K. M. Adams (Eds.), Neuropsychological assessment of neuropsychiatric disorders (1st ed., pp. 100-120). New York: Oxford University Press.

Hedges, L. V., Laine, R. D., \& Greenwald, R. (1994). Does money matter? A meta-analysis of studies of the effects of differential school inputs on student outcomes. Educational Researcher, 23(3), 5-14.

Helms, J. E. (1992). Why is there no study of cultural equivalence in standardized cognitive ability testing? American Psychologist, 47, 1083-1101.

Hilliard, A. G. (1979). Standardization and cultural bias impediments to the scientific study and validation of "intelligence."Journal of Research and Development in Education, $12,47-58$.

Kaplan, E., Goodglass, H., \& Weintraub, S. (1983). Boston Naming Test. Philadelphia: Lea \& Febiger.

Kaufman, J. S., Cooper, R. S., \& McGee, D. L. (1997). Socioeconomic status and health in blacks and whites: The problem of residual confounding and the resilience of race. Epidemiology, $8,621-628$.

Kennepohl, S., Shore, D., Nabors, N., \& Hanks, R. (in press). African American acculturation and neuropsychological test performance following traumatic brain injury. Journal of the International Neuropsychological Society.

Klineberg, O. (1935). Race differences. New York: Harper \& Brothers.

Klusman, L. E., Moulton, J. M., Hornbostle, L. K., Picano, J. J., \& Beattie, M. T. (1991). Neuropsychological abnormalities in asymptomatic HIV seropositive military personnel. Journal of Neuropsychological and Clinical Neurosciences, 3, 422-428.

Landrine, H., \& Klonoff, E. A. (1994). The African American Acculturation Scale: Development, reliability, and validity. Journal of Black Psychology, 20, 104-127.

Landrine, H., \& Klonoff, E. A. (1995). The African American Acculturation Scale II: Cross-validation and short form. Journal of Black Psychology, 21, 124-152.

Lezak, M. D. (1995). Neuropsychological assessment (3rd ed.). New York: Oxford University Press.

Loewenstein, D. A., Arguelles, T., Arguelles, S., \& Linn-Fuentes, P. (1994). Potential cultural bias in the neuropsychological assessment of the older adult. Journal of Clinical and Experimental Neuropsychology, 16, 623-629.

Lucas, J. A. (1998). Acculturation and neuropsychological test performance in elderly African Americans. Journal of the International Neuropsychological Society, 4, 77.

Manly, J. J., \& Jacobs, D. M. (2001). Future directions in neuropsychological assessment with African Americans. In F. R. Ferraro (Ed.), Minority and cross-cultural aspects of neuropsychological assessment (pp. 79-96). Lisse, Netherlands: Swets \& Zeitlinger.

Manly, J. J., Jacobs, D. M., Sano, M., Bell, K., Merchant, C. A., Small, S. A., et al. (1998a). African American acculturation and neuropsychological test performance among nondemented community elders. Journal of the International Neuropsychological Society, 4, 77.

Manly, J. J., Jacobs, D. M., Sano, M., Bell, K., Merchant, C. A., Small, S. A., et al. (1998b). Cognitive test performance among 
nondemented elderly African Americans and Whites. Neurology, 50, 1238-1245.

Manly, J. J., Jacobs, D. M., Sano, M., Bell, K., Merchant, C. A., Small, S. A., et al. (1999). Effect of literacy on neuropsychological test performance in nondemented, education-matched elders. Journal of the International Neuropsychological Society, 5, 191-202.

Manly, J. J., Jacobs, D. M., Touradji, P., Small, S. A., \& Stern, Y. (2002). Reading level attenuates differences in neuropsychological test performance between African American and White elders. Journal of the International Neuropsychological Society, 8, 341-348.

Manly, J. J., Miller, S. W., Heaton, R. K., Byrd, D., Reilly, J., Velasquez, R. J., et al. (1998). The effect of African American acculturation on neuropsychological test performance in normal and HIV positive individuals. Journal of the International Neuropsychological Society, 4, 291-302.

Margo, R. A. (1990). Race and schooling in the South, 1880-1950: An economic history. Chicago: University of Chicago Press.

Mattis, S. (1976). Mental status examination for organic mental syndrome in the elderly patient. In L. Bellak \& T. B. Karasu (Eds.), Geriatric psychiatry (pp. 77-121). New York: Grune \& Stratton.

Mercer, J. R. (1974). Labeling the mentally retarded: Clinical and social system perspectives on mental retardation. Berkeley: University of California Press.

Moyerman, D. R., \& Forman, B. D. (1992). Acculturation and adjustment-a meta-analytic study. Hispanic Journal of Behavioral Sciences, 14, 163-200.

Nabors, N. A., Evans, J. D., \& Strickland, T. L. (2000). Neuropsychological assessment and intervention with African Americans. In E. Fletcher-Janzen, T. L. Strickland, \& C. Reynolds (Eds.), Handbook of cross-cultural neuropsychology (pp. 31-42). New York: Kluwer Academic.

Negy, C., \& Woods, D. J. (1992). The importance of acculturation in understanding research with Hispanic-Americans. Hispanic Journal of Behavioral Sciences, 14, 224-247.

Neisser, U., Boodoo, G., Bouchard, T. J. J., Boykin, A. W., Brody, N., Ceci, S. J., et al. (1996). Intelligence: Knowns and unknowns. American Psychologist, 51, 77-101.

O'Neill, J. (1990). The role of human capitol in earning differences between Black and White men. Journal of Economic Perspectives, 4, 25-45.

Padilla, A. M. (1980). Acculturation: Theory, models, and some new findings. Boulder, $\mathrm{CO}$ : Westview for the American Association for the Advancement of Science.

Powell, W. A., Tuchin, S. R., Touradji, P., \& Manly, J. J. (2000). African American acculturation among nondemented elders. $\mathrm{Pa}-$ per presented at the annual meeting of the American Psychological Association, Washington, DC.

Price-Williams, D. R., \& Ramirez, M. (1977). Divergent thinking, cultural deficiencies and civilizations. Journal of Social Psychology, 103, 3-11.

Rosen, W. (1981). The Rosen Drawing Test. Bronx, NY: Veterans Administration Medical Center.

Shade, B. J. (1991). African American patterns of cognition. In R. L. Jones (Ed.), Black psychology (3rd ed., pp. 231-247). Berkeley, CA: Cobb \& Henry Publishers.

Smedley, A. (1999). Race in North America: Origin and evolution of a worldview. (2nd ed.) Boulder, $\mathrm{CO}$ : Westview.

Stern, Y., Andrews, H., Pittman, J., Sano, M., Tatemichi, T., Lantigua, R., et al. (1992). Diagnosis of dementia in a heterogeneous population. Development of a neuropsychological paradigm-based diagnosis of dementia and quantified correction for the effects of education. Archives of Neurology, 49, 453-460.

Terrell, F., Terrell, S. L., \& Taylor, J. (1981). Effects of race of examiner and cultural mistrust on the WAIS performance of Black students. Journal of Consulting and Clinical Psychology, 49, 750-751.

Torrance, E. P. (1971). Are the Torrance Tests of Creative Thinking biased against or in favor of "disadvantaged" groups? Gifted Child Quarterly, 15, 75-80.

van de Vijver, F. (1997). Meta-analysis of cross-cultural comparisons of cognitive test performance. Journal of Cross-Cultural Psychology, 28, 678-709.

Wechsler, D. (1981). Wechsler Adult Intelligence Scale-Revised. New York: Psychological Corporation.

Welsh, K. A., Fillenbaum, G., Wilkinson, W., Heyman, A., Mohs, R. C., Stern, Y., et al. (1995). Neuropsychological test performance in African American and white patients with Alzheimer's disease. Neurology, 45, 2207-2211.

Wilkinson, G. S. (1993). Wide Range Achievement Test 3-Administration manual. Wilmington, DE: Jastak Associates.

Wilkinson, D. Y., \& King, G. (1987). Conceptual and methodological issues in the use of race as a variable: Policy implications. Milbank Quarterly, 65, 56-71.

Williams, R. L. (1971). Abuses and misuses in testing black children. Counseling Psychologist, 2, 62-73.

Williams, R. L. (1974). Scientific racism and IQ: The silent mugging of the black community. Psychology Today, 7, 32-41.

Wong, T. M., Strickland, T. L., Fletcher-Janzen, E., Ardila, A., \& Reynolds, C. R. (2000). Theoretical and practical issues in the neuropsychological assessment and treatment of culturally dissimilar patients. In E. Fletcher-Janzen, T. L. Strickland, \& C. R. Reynolds (Eds.), Handbook of cross-cultural neuropsychology (pp. 3-18). New York: Kluwer Academic. 
Copyright of Applied Neuropsychology is the property of Lawrence Erlbaum Associates and its content may not be copied or emailed to multiple sites or posted to a listserv without the copyright holder's express written permission. However, users may print, download, or email articles for individual use. 